\section{Praxiserprobung}

Im Rahmen des Projektes angewandte Ökologie (PAÖ) fördert die Landesanstalt für Umweltschutz Baden-Württemberg (Karlsruhe) ein Forschungsvorhaben zur Entwicklung und Praxiserprobung des betrieblichen Stoff- und Energieflußmanagements. Durchgeführt wird das Projekt vom Institut für Management und Umwelt (Augsburg). Die Praxiserprobung erfolgt bei dem Arzneimittelhersteller Merckle/ratiopharm (Ulm), der bereits mit allen drei Produktionsstandorten gemäß der EG-ÖkoAudit-Verordnung begutachtet ist. Das Projekt wird Ende des Jahres 1997 abgeschlossen sein. Projektziele sind neben der Verbesserung der Stoff- und Energieflußtransparenz, die Entwicklung der Organisation und die Entwicklung von kostensenkenden und umweltentlastenden Maßnahmen. Die Ergebnisse werden Mitte nächsten Jahres in Form eines Leitfadens veröffentlicht.

\section{Anmerkungen}

(1) Blick durch die Wirtschaft vom 16.12.1994 sowie Gottschall, Dieter: Grüne Welle. In: manager magazin 6/1994, S. 156., sowie

le Maire, Gunther: Die Kunert-0̈kobilanz. In: Umweltqualitätsziele von Unternehmen und ihre Durchsetzung, Schriftenreihe des IÖW 60/93, Berlin 1993; S. 102.

(2) Strobel, Markus/Wagner, Bernd: Strukturierung und Entwicklung der betrieblichen Stoff- und Energieflüsse, in: Fischer, Hartmut/Wucherer, Christion/Wagner, Bernd/Burschel, Carlo: Umweltkostenmanagement, München 1997, S. 57., sowie

KUNERT AG et. al.: Modellprojekt Umweltkosten-Management (AbschluBbericht), Immenstadt 1995.

\section{Die Autoren}

Markus Strobel ist Geschäftsführer des Instituts für Management und Umwelt (imu).

Stefan Enzler ist beim imu als wissenschaftlicher Mitarbeiter beschäfligt.

Kontakt: Institut für Management und Umwelt, Gratzmülersirafle 3, 86150 Augsburg

Referenzmodell für DV-gestütztes betriebliches Umweltmanagement

\title{
Fortschritte durch ECO-Integral
}

\begin{abstract}
Wie können das Umweltmanagement und die Integration von Ökonomie und Ökologie erkennbar gestärkt werden? Das Referenzmodell ECO-Integral will hierzu einen Beitrag leisten. Durch dieses neu entwickelte Modell soll die Integration wesentlicher Instrumente des betrieblichen Umweltmanagements in ein Gesamtkonzept und in Standardsoftware vorangetrieben werden.
\end{abstract}

Von Georg Dold, Stefan Enzler, Hartmut Fischer, Astrid Hoffmann, Helmut Krcmar, Martin Kreeb, Michael Oettinger, Wolfgang Scheide, Eberhard K. Seifert, Markus Strobel, Bernd Wagner

$\mathrm{I}$ m Sinne einer stärkeren Einbindung von „begin-of-the-pipe“-Technologien in organisatorische Prozesse ist es die zentrale Aufgabe des Umweltmanagements, die Stoff- und Energieflüsse effizient und ökologisch zu gestalten. Dazu muß das Umweltmanagement in die Entscheidungsprozesse der Organisationseinheiten (Einkauf, Produktentwicklung, Produktion, Rechnungswesen und Marketing) integriert werden. Bisherige Erfahrungen von Pilotunternehmen mit Umweltmanagement dieser modernen Form führen zur Aussage „Umweltmanagement kann Kosten senken und Umwelt entlasten“. Grundlage dafür ist eine hohe Transparenz der Mengen, Kosten und Umweltwirkungen der betrieblichen Stoff- und Energieflüsse. Erst durch effiziente Informationsversorgung können erprobte und nutzbringende Instrumente des Umweltmanagements ihre Leistungsfähigkeit entfalten (z. B. Umweltkostenrechnung, Kennzahlenbildung, Ökobilanzierung). Somit sind die Informationssysteme das Bindeglied zwischen Stoffund Energieflüssen sowie Entscheidungsprozessen (vgl. Abbildung 1).

\section{Projektablauf und Vorgehensweise}

Im Projekt ECO-Integral erarbeiten mit Förderung der Deutschen Bundesstiftung Umwelt (DBU) die Partner Universität Hohenheim, Kienbaum Unternehmensberatung, Institut für Management und

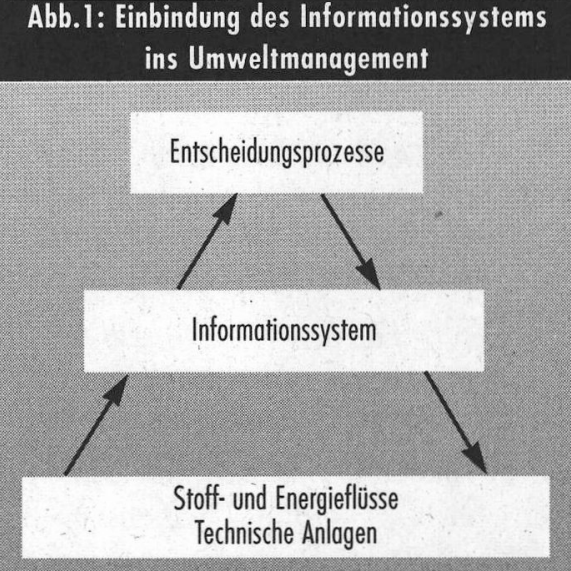

Umwelt sowie das Wuppertal-Institut gemeinsam ein Referenzmodell für DVgestütztes Umweltmanagement. Das Projekt wird in drei Phasen durchgeführt (vgl. Abbildung 2). Zunächst wird das Referenzmodell ECO-Integral ,am grünen Tisch" entwickelt und an drei Industriestandorten evaluiert und weiterentwickelt. In einer zweiten Phase soll mit Software-Herstellern deren Standardsoftware nach ECO-Integral modifiziert und in Pilotinstallationen in der Industrie erprobt werden. Zuletzt soll das Referenzmodell als Softwarelösung umgesetzt und von den beteiligten SoftwareHerstellern in den Markt getragen werden.

In drei Pilotunternehmen wird die aktuelle Version des Referenzmodells derzeit evaluiert. Dieses beschreibt die Umweltmanagementanforderungen an ein Informationssystem in strukturierter Form. Als Modellierungswerkzeug wurde ARIS verwendet (Architektur integrierter Informationssysteme, vgl. Scheer 1992). ARIS ist ein offener Modellierungsstandard und legt für softwaretechnische Zwecke vier Sichten auf ein Informati- 
Abb.2: Vorgehen im ECO-Integral Projekt

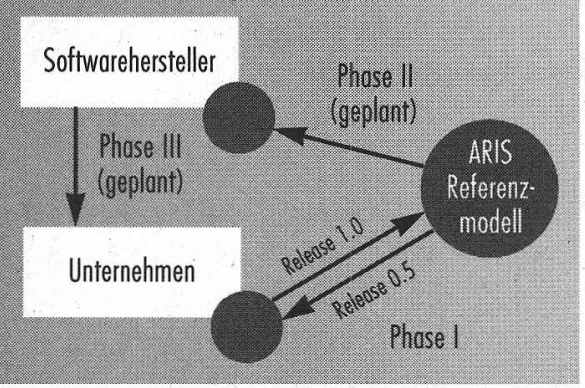

onssystem: Daten, Funktionen, Steuerung und Organisation. Die Integration dieser Sichten erlaubt eine Beschreibung aller wichtigen Aspekte eines Informationssystems. Wegen der von ARIS geforderten formalen, DV-nahen Beschreibung eines Fachgebietes sind verwendete Begriffe klar zu definieren. Beim hier modellierten Fachgebiet „Umweltmanagement" wurde sichtbar, daß viele Begriffe derzeit mit unterschiedlichen Inhalten belegt sind (z.B. „Öko-Controlling“). Daher war es notwendig, grundlegende Begriffe des Umweltmanagements auf ein gemeinsames Verständnis festzulegen. Das Referenzmodell liegt mittlerweile als Funktionsbaum mit drei Hauptzweigen vor: „Führungsfunktionen unterstützen“, „Instrumente einsetzen“ und „Datenbasis erstellen“. Weiterhin sind wesentliche Abläufe als EreignisProzeßketten (EPKs) modelliert und ein Datenmodell (ERM) erstellt worden. Über die Inhalte des Modells berichtet das Folgekapitel.

\section{Referenzmodell ECO-Integral}

Das Referenzmodell ECO-Integral besteht aus einer gemeinsamen Datenbasis und einer Reihe von Instrumenten des Umweltmanagements, die auf diese Datenbasis zugreifen.

Die Datenbasis muß alle relevanten Stoff- und Energiebewegungen in einem einheitlichen Format enthalten. Dazu bietet sich die. Datei an, in der bereits große Bewegungsmengen geführt werden. In SAP R/2 ist dies z. B. die Belegdatei. Sie ist um Bewegungen von Energien (Strom, Wärme, Wasser) und Reststoffen (Abfall, Abwasser und Abluft) zu ergänzen. In der Unternehmenspraxis geschieht dies meist nicht. Viele der zusätzlich abgelegten Bewegungen sind aus
Wirtschaftlichkeitsgründen zu errechnen (statt Ist-Erfassung am Entstehungs-/Verbrauchsort). ECO-Integral enthält dafür umfangreiche Errechnungsfunktionen. Beispielsweise können stückvariable Reststoffmengen als negative Positionen mit ergänzenden Kennungen in der Stückliste verankert werden. Über die retrograde Entnahme können aus den Stücklistendaten dann Abfallmengen ermittelt werden. Für die Ablage von Bewegungen in einem einheitlichen Format ist vorgesehen, alle Stoffe und Energien in einer übergreifenden Materialstruktur abzulegen sowie alle Quellen und Senken von Stoff- und Energieflüssen in einer übergreifenden Mengenstellenstruktur (analog der Kostenstellenstruktur) abzulegen.

Das Instrument Umweltkostenrechnung unterstuitzt die Erstellung von Berichten nach $\S 15$ UStatG, die verursachungsgerechtere Zuordnung von „end-of-thepipe"-Kosten sowie die Reststoffkostenrechnung. Die Reststoffkostenrechnung weist die vollen Kosten von Abfall, Abwasser und Abluft (in der Regel fünf bis 15 Prozent der Gesamtkosten) aus und eröffnet damit den Zugang zu nennenswerten Kostensenkungsreserven und Umweltentlastungen. Aufbauend auf der mit ECO-Integral verbesserten Mengentransparenz werden einzelnen Reststoffströmen Kostenelemente ganz oder anteilig zugeordnet. Die erforderlichen Zuordnungen werden aus Primärbuchungen zu Mengen, Kosten und Leistungen, aus dauerhaft hinterlegten Zuordnungen oder aus aktuellen Schlüsselmengen abgeleitet. Relevante umweltinduzierte Kosten lassen sich somit Kostenstellen und Kostenträgern verursachungsgerechter zuweisen. In einer Nebenrechnung können diese Kosten flexibel ausgewertet (z. B. nach Einsatzmaterialien, Fertigungsprozessen, verursachenden Produkten, Zeiträumen) und zu Kennzahlen für das Umweltkostenmanagement verdichtet werden.

Das Umweltrecht stellt hohe Anforderungen an Industrieunternehmen: Eine wachsende Anzahl von stoffflußbezogenen Berichten ist zu erstellen, und es sind viele Grenz- und Schwellenwerte zu überwachen und einzuhalten. Die Novellierung des Umweltstatistik-Gesetzes (UStatG) vom 21.09.1994 und zusätzliche Vorgaben (inter-)nationaler Institutionen und Ämter sowie der Normungsgremien DIN und ISO bedeuten einen zusätzlichen $\mathrm{zu}$ befriedigenden Informationsbedarf für die Unternehmen, der in ECO-Integral als Micro-Macro-Link berücksichtigt wird. Die erforderliche Datenerhebung bedeutet einen großen Zusatzaufwand. Das Referenzmodell ECO-Integral ist deshalb so ausgelegt, daß die für die Erstellung von stoff- und energieflußbezogenen Berichten an Behörden aus den Bereichen Abfall, Abwasser, Abluft und Gefahrstoffe notwendigen Daten DV-gestützt bereitgestellt werden können. Außerdem wird die automatische Überwachung der Einhaltung umweltrechtlicher und in Genehmigungsbescheiden vorgegebener Grenzwerte unterstützt. Ziel dabei ist es, den Aufwand für die Erstellung von umweltrechtlich erforderlichen Berichten erheblich zu senken und die Qualität der Daten für die rechtskonforme Steuerung des betrieblichen Umweltmanagements, die Abwehr von Haftungsansprüchen und die Planungs- und Überwachungsaufgaben der Behörden zu steigern. Bei den dafür notwendigen Daten handelt es sich in der Regel um aggregierte Stoffflüsse in einem Zeitraum (z. B. Abfallbilanz), Bestandsmengen $\mathrm{zu}$ einem Zeitpunkt (Höchstmengen eines gelagerten Gefahrstoffes) und Entstehungsorte bzw. Verbringungsorte bestimmter Materialien. Durch die integrierte ECO-IntegralDatenbasis müssen die notwendigen Grunddaten nur einmal eingegeben werden und können automatisiert für die verschiedenen Zwecke zusammengestellt werden.

Die Unterstützung von Umweltmanagementsystemen zielt auf die EG-ÖkoAudit-Verordnung (EMAS) und die ISO 14001 bzw. 14031. Ansatzpunkte für kontinuierliche Verbesserungen im Umweltschutz sind die Kenntnis der betrieblichen Stoff- und Energieflüsse und zugehörige Möglichkeiten für kontrollierte Veränderungen. Vor diesem Hintergrund unterstützt das Referenzmo- 
dell ECO-Integral folgende Instrumente: Ermittlung von Umweltkennzahlen, Umweltprogramm-Verwaltung, Soll-IstAbgleiche und Berichtserstellung (Umwelterklärung/Umweltbericht). Das Instrument Umweltkennzahlen zeigt in komprimierter Form die Umweltauswirkungen des Unternehmens auf und ermöglicht eine Quantifizierung der Umweltziele. Diese Ziele können durch das Instrument Umweltprogramm-Verwaltung mit Maßnahmen, Fristen, Verantwortlichkeiten und weiteren Bezugsgrößen versehen und somit einer effizienten Umsetzung entgegengeführt werden. Im Rahmen des Soll-IstAbgleichs wird der Zielerreichungsgrad berechnet und damit eine wirksame Erfolgskontrolle durchgeführt. Bei Bedarf ist eine Anpassung der Umweltziele möglich. Die Umweltleistung eines Unternehmens kann somit meßbar gemacht und einer kontinuierlichen Verbesserung zugeführt werden. Weiterhin unterstützt ECO-Integral die Erstellung des stoff- und energieflußbezogenen Datenteils von Umwelterklärungen bzw. Umweltberichten

Das Instrument Ökobilanz ist genau abzugrenzen, da in der wissenschaftlichen Diskussion und in der praktischen Anwendung unterschiedliche Auffassungen über die inhaltliche Ausgestaltung von Ökobilanzen bestehen. Einfache betriebliche Input-Output-Analysen und komplexe Produktlebensweganalysen werden gleichermaßen als Ökobilanz bezeichnet. Um diesen Unklarheiten vorzubeugen, enthält das Referenzmodell ECO-Integral drei unabhängige Instrumente, die gemeinsam das übergeordnete Instrument Ökobilanz umfassen. In ECO-Integral sind modelliert:

- Stoff- und Energiebilanzen für Standorte und Prozesse. Hier werden Stoffund Energieflüsse eines Bilanzobjektes (Unternehmen, Standorte, Prozesse) strukturiert gegenübergestellt.

- Interne Stoff- und Energiebilanzen für Produkte. Diese Bilanzen beinhalten die von einem Produkt innerhalb eines Unternehmens verursachten Stoff- und Energieflüsse.
- Wirkungsanalysen und Bewertungsverfahren, mit denen die aggregierten Stoff- und Energieflüsse analysiert und bewertet werden können.

Zur Erstellung der Stoff- und Energiebilanzen werden zuerst der Bilanzraum abgegrenzt und die Bilanzperiode festgelegt. Anschließend werden die Bewegungsdaten nach einer vorgegebenen oder vom Anwender definierten Bilanzstruktur automatisch aus der gemeinsamen Datenbasis aggregiert. Zur Bewertung der Umweltauswirkung der ermittelten Stoff- und Energieflüsse stehen unterschiedliche Verfahren zur Auswahl.

\section{- Praxiserfahrungen und Fazif}

Innerhalb des Projekts ECO-Integral werden drei Firmenprojekte durchgeführt. Dabei handelt es sich um jeweils einen Standort der Herlitz PBS AG (Papier-, Büro- und Schreibwaren; stücklistenorientiert), Novartis Pharma GmbH (Pharmazeutika; rezepturorientiert) und der Festo KG (pneumatische Steuerungselemente; stïcklistenorientiert). Folgende vorläufige Ergebnisse zeichnen sich aus der Zusammenarbeit mit diesen produzierenden Unternehmen ab:

- ECO-Integral hat ein Angebot von Funktionen, aus dem für jedes der Unternehmen ein attraktives Konzept zusammengestellt werden konnte. Auf unmittelbare Resonanz treffen die folgenden Instrumente: die Umweltkostenrechnung, von deren Einführung klare Kostenvorteile erhofft werden (Senkung der Reststoff- und Qualitätskosten); die DVgestützte Erstellung von Betriebsbilanzen wird gewünscht, um den Datenerfassungsaufwand zu senken; Unterstützung von EMAS/ISO 14000ff und umweltrechtlichen Anforderungen (z. B. UStatG) scheint gerade im Zusammenhang mit der Ablösung bisheriger Anwendungen interessant.

- Reststoffe machen einen erheblichen Teil des Materialflusses aus. Sie werden jedoch mengenmäßig nicht ausreichend erfaßt. ECO-Integral leistet die datenseitige und operative Integration der Reststoffe. Dies stärkt den Status und Einfluß des Umweltmanagements erkennbar.
Zudem wird so ein direkter Beitrag zum Kosten-, Qualitäts- und Logistikmanagement geleistet.

- Bei der Umsetzung von ECO-Integral sollten in den Unternehmen „Datenlieferanten“, „Datenverarbeiter", „Datennutzer" und ein Mitglied der Geschäftsleitung beteiligt sein, um ein abgestimmtes Gesamtkonzept und seine Durchsetzung zu gewährleisten.

- Ein wichtiger Teil der für ECO-Integral erforderlichen Funktionalitäten ist in großen Standard-Softwaresystemen schon heute teilweise verfügbar. Größere Anpassungen dieser Anwendungen werden erforderlich, um neue operative Funktionen bereitzustellen (z.B. für die Abfallwirtschaft), komplexe Stoffumwandlungsprozesse abzubilden und Berichtsgeneratoren verfügbar $\mathrm{zu}$. machen.

Zusammenfassend kann festgehalten werden, daß das Referenzmodell ECOIntegral einen Beitrag dazu leisten kann, die Integration wesentlicher Instrumente des betrieblichen Umweltmanagements in ein Gesamtkonzept und in Standardsoftware voranzutreiben. Durch Anwendung von ECO-Integral werden das Umweltmanagement sowie die Integration von Ökonomie und Ökologie erkennbar gestärkt. Weiterer Handlungsbedarf besteht nun darin, ECO-Integral zu implementieren und zu erproben. Zudem kann das Referenzmodell um zusätzliche Einzelfunktionen des Umweltmanagements ergänzt werden.

\section{Die Autoren}

H. Krcmar, G. Dold, W. Scheide, M. Oettinger, Universitäı Hohenheim, Institul für Beiriebswirtschaftslehre, Lehrstuhl für Wirtschaftsinformatik (510H), 70593 Stuitgart;

B. Wagner, M. Strobel, A. Hoffmann, S. Enzler, Insiitui für Management und Umwelt, Graizmüllersirafle 3, 86150 Augshurg;

H. Fischer, Kienbaum Unternehmensberatung $\mathrm{GmbH}$, Bleibtreustrafle I, 10623 Berlin;

E. K. Seifert, M. Kreeb, Wuppertal Institut für Klima, Umwelt und Energie GmbH, Postfach 100480 , 42004 Wuppertal. 
(c) 20I0 Authors; licensee IÖW and oekom verlag. This is an article distributed under the terms of the Creative Commons Attribution Non-Commercial No Derivates License (http://creativecommons.org/licenses/by-nc-nd/3.o/), which permits unrestricted use, distribution, and reproduction in any medium, provided the original work is properly cited. 